\title{
„Pain is inevitable, suffering is optional": Fallkonzeption in der Kognitiven Verhaltenstherapie
}

\author{
Horst Mitmansgruber · Andrea Fahlböck · Alexander Fink · Liselotte Mäni Kogler • Ilse Müller • \\ Annamaria Painold · Christa Streicher-Pehböck · Gerald Gatterer
}

Zusammenfassung Anhand eines praktischen Beispiels werden der diagnostische Prozess und die Fallkonzeption in der Kognitiven Verhaltenstherapie präsentiert. Die Diagnose stellt einen wichtigen Ausgangspunkt für die theoretische Konzeptualisierung und praktische Behandlung mit evidenz-basierten therapeutischen Methoden dar. Die Unterschiede zwischen Patient*innen und ihre idiosynkratische Beziehungsgestaltung in der Therapie erfordern allerdings eine individuelle Fallkonzeption. In einem Diathese-Stress-Modell werden als „Arbeitshypothese“ (1) lebensgeschichtliche Ereignisse und Bedingungen (Stressoren, Traumata etc.) identifiziert und beschrieben, die (2) langjährige (auch interaktionelle) Denkund Verhaltensmuster als Vulnerabilität nach sich gezogen haben. Zusammen mit (3) Auslösern (aktuellen schema-aktivierenden Situationen) bringen sie (4) die Symptomatik als Muster von Gedanken, Gefühlen und Verhalten hervor. Diese wird (5) durch

H. Mitmansgruber $(\bowtie) \cdot$ A. Fahlböck · A. Fink · L. Mäni

Kogler · I. Müller · A. Painold · C. Streicher-Pehböck ·

G. Gatterer

Ausbildungsverantwortliches und Anerkennungsgremium

(AVNG) der AVM, Arbeitsgemeinschaft

für Verhaltensmodifikation Österreich,

Paris-Lodron-Straße 32, 5020 Salzburg, Österreich

horst.mitmansgruber@i-med.ac.at

\section{H. Mitmansgruber}

Universitätsklinik für Medizinische Psychologie, Medizinische Universität Innsbruck,

Speckbacherstraße 23, 6020 Innsbruck, Österreich

\section{A. Fahlböck}

Institut für neuropsychologische Rehabilitation, Widmanngasse 43, 9500 Villach, Österreich

\section{A. Fink}

Zentrum Psychotherapie, Berchtesgadner Straße 35b, 5020 Salzburg, Österreich dysfunktionale Bewältigungsversuche aufrechterhalten und in „Teufelskreisen“ stabilisiert. Kernstück der Diagnostik sind sog. horizontale und vertikale Verhaltensanalysen, die in ein „hypothetisches Bedingungsmodell“ münden. Dieses Modell stellt ein Gesamtbild der Problematik und der interaktionellen Muster auch unter Berücksichtigung der Stärken und Ressourcen einer Person dar. Klinisch nützlich ist ein Fallkonzept, wenn es kollaborativ (in aktiver Mitarbeit von Patient*innen) erstellt und diskutiert wurde und die unmittelbare Ableitung von gemeinsamen therapeutischen Ansatzpunkten und Zielen erlaubt. Gleichzeitig weist es auf mögliche Schwierigkeiten von Patient*innen im therapeutischen Prozess hin (d.h. eventuelle motivationale Barrieren und „Widerstände“). Zentrale Elemente unseres Vorgehens im Entwickeln einer kognitiv-verhaltenstherapeutischen

\section{Mäni Kogler · I. Müller \\ Institut für Psychosomatik und Verhaltenstherapie,} Alberstraße 15, 8010 Graz, Österreich

\section{A. Painold}

Universitätsklinik für Psychiatrie und Psychotherapeutische

Medizin, Medizinische Universität Graz,

Auenbruggerplatz 31, 8036 Graz, Österreich

\section{Streicher-Pehböck}

Psychologische Studierendenberatung,

Altenbergerstraße 69, 4040 Linz, Österreich

\section{G. Gatterer}

Sozialtherapeutisches Zentrum, Therapiezentrum Ybbs, Persenbeugerstraße 1-3, 3370 Ybbs/Donau, Österreich 
Fallkonzeption illustrieren wir mit dem Fall einer 34jährigen depressiven Patientin.

Schlüsselwörter Kognitive Verhaltenstherapie · Individualisierte Fallkonzeption · Fallformulierung · Hypothetisches Bedingungsmodell · Horizontale und vertikale Verhaltensanalyse

\section{"Pain is inevitable, suffering is optional": Case conceptualization in cognitive-behavioral therapy}

Summary Using the example of a depressive patient, the process of diagnostics and case formulation in cognitive behavioral therapy is illustrated. Diagnosis is an important starting point for theoretical conceptualization and the treatment with evidence-based methods. However, the differences between patients and their idiosyncratic relationship with the therapist require individualized case formulations. A diathesis-stress-model is the basis for a "working hypothesis" that identifies (1) biographical events and factors (stressors, traumata, etc.) which (2) result in longlasting patterns of thought and behavior (also interactional) that comprise a central vulnerability of the person. (3) Triggering events activate the vulnerability and result in (4) symptoms as patterns of thoughts, emotions, and behaviors which (5) are maintained by dysfunctional coping behavior and "vicious circles". Central diagnostic approaches are systematic analyses of behavior that result in a hypothetical working model. This is an overall theory in a nutshell for the patients' problems and interaction patterns including strenghts and resources. A case formulation has clinical utility if it is developed and discussed collaboratively and allows the formulation of consensual therapeutic goals. At the same time, it points out possible difficulties of the patient in the process of therapy (i.e. possible motivational barriers and "resistance"). We illustrate core processes in the development of a cognitive-behavioral case coneptualization with the case example.

Keywords Cognitive-behavioral therapy • Individualized case conceptualization · Case formulation · Hypothetical working model . Horizontal and vertical behavior analysis

\section{Einführung}

Wie wissen wir, was wir wann mit unseren Patient*innen in der Therapie tun sollen? Wie können wir in einer mitunter sehr komplexen Situation planvoll vorgehen? In der Kognitiven Verhaltenstherapie (KVT) haben standardisierte Therapieprogramme und individualisierte Fallkonzeptionen eine gleichermaßen große Tradition (Bruch 2015; Sturmey 2009). Auf der einen Seite hat die empirische Untersuchung von störungsspezifischen Therapiemanualen mit ih- ren prototypischen Fallkonzeptionen die Wirksamkeit von Psychotherapie eindrucksvoll belegen können (National Institute of Clinical Excellence, NICE 2020; American Psychological Association 2020). Auf der anderen Seite sind es ganz individuelle Verhaltensanalysen, mit denen die Probleme von Patient*innen über vorausgehende und nachfolgende Bedingungen und kognitive Bewertungsprozesse konzeptualisiert werden (Kanfer et al. 2012; Beck und Haigh 2014). Daher auch das Zitat im Titel: Nicht der Schmerz ist das Problem, es ist seine Bewertung, die daraus Leiden macht (die Quelle des Zitats ist nicht exakt zuordenbar, es stammt offenbar aus dem Buddhismus). Aktuell werden in einer „evidence-based practice“ (Dimidjian 2019) die zentralen Vorteile beider Herangehensweisen genützt: sich klar an der empirischen Evidenz in einem Störungsbereich orientieren und gleichzeitig den besonderen Gegebenheiten der Lebens- und Behandlungssituation von Patient*innen Rechnung tragen (Lilienfeld et al. 2019).

Die individualisierte Fallkonzeption „beschreibt Symptome, Störungen und Probleme von Patient*innen und postuliert Mechanismen, die diese Probleme verursachen, sowie Auslöser und Wurzeln dieser Mechanismen. Die Fallkonzeption verbindet diese Elemente zu einem kohärenten Ganzen“ (Persons et al. 2019, S. 148, dt. Übers. HM). Sie hilft hier auch beim Treffen komplexer klinischer Entscheidungen (Eells 2007, 2015). Störungsspezifische Therapiemanuale liefern kaum Informationen dazu, wie Patient*innen behandelt werden sollen, die (1) mehrere Probleme in die Therapie mitbringen (z.B. Angst, Depression und Alkoholismus). Sie liefern (2) keine Information zu einer Vielzahl von Entscheidungen, die im therapeutischen Prozess getroffen werden müssen (z. B. Ist es sinnvoll, eine Patient*in, die ungeplant weggeblieben ist, nach drei Wochen zu kontaktieren oder eben nicht?). Zudem existieren (3) für einige Problemfelder keine evidenz-basierten therapeutischen Methoden (z.B. hochfunktionaler Autismus bei Erwachsenen, dissoziative Störungen). Schließlich liefern Therapiemanuale (4) häufig keine Informationen dazu, wie mit Non-adherence oder Schwierigkeiten in der therapeutischen Beziehung bzw. Nicht-Ansprechen auf die Therapie umgegangen werden soll (Key und Bieling 2014; Persons 2008).

In diesen Situationen helfen individualisierte Fallkonzeptionen. Hierzu liegt eine Reihe von ausgezeichneten Modellen vor, wie wir die Informationen von unseren Patient*innen verstehen und organisieren können (Bartling et al. 2016; Beck 2013; Bruch 2015; Dobson und Dobson 2017; Kanfer et al. 2012; Kuyken et al. 2009; Ledley et al. 2018; Needleman 1999; Nezu et al. 2004; Persons 2008; Reinecker 2015; Sturmey 2009; Tarrier 2006). Wir möchten die gemeinsame Basis dieser Modelle beschreiben und diese anhand einer 34-jährigen Patientin illustrieren, die wir in Teilen und Ausschnitten (nicht immer chronologisch) präsentieren. 
Frau S. (34; Daten geändert) kommt nach zweimonatigem Krankenstand auf Empfehlung der Psychiaterin. Sie leide seit 8 Monaten an zunehmender und relativ durchgängiger Niedergeschlagenheit und Freudlosigkeit. Sie habe sich deutlich zurückgezogen und habe eine Reihe von Aktivitäten mit Freunden wie auch Sport reduziert. Sie fühle sich ständig müde, hätte sich nur mühsam morgens hochgekämpft und wäre in der Arbeit unkonzentriert, überfordert und weinerlich gewesen. Sie hätte abends weiter gegrübelt und könne weder ein- noch durchschlafen. Der Appetit sei unverändert. Sie fühle sich hilflos und einsam. Suizidalität sei kein Thema. Medizinisch sei sie abgeklärt und sie habe ein hilfreiches schlafanstoßendes Antidepressivum erhalten. Mehrere Panikattacken in der Arbeit mit Atemnot und Angst zu ersticken hätten sie schließlich bewogen in Krankenstand zu gehen. Seither hätte sich die Panik bzw. die Angst vor den Attacken gebessert. Frau S. habe eine ähnliche deprimierte Phase bereits vor 7 Jahren nach der Trennung von ihrem damaligen Freund erlebt, die damals 3-4 Monate angedauert habe.

\section{Die ICD-Diagnose als vorläufiger Start für therapeutische Überlegungen}

Die Patientin leidet an einer rezidivierenden depressiven Störung, gegenwärtig mittelgradige Episode. Warum eine Diagnose, wenn eine individualisierte Fallkonzeption ohnehin auf den besonderen Menschen zugeschnitten wird? Die krankheitswertige Störung ermöglicht ihr zunächst den offiziellen Eintritt in das Gesundheitssystem und erleichtert die Kommunikation unter Behandler*innen. Wichtiger im aktuellen Zusammenhang ist allerdings erstens, dass zurzeit der überwiegende Teil der Literatur zu theoretischen Modellen und Behandlungseffektivität über die Diagnose organisiert ist und so gefunden werden kann. Zweitens unterstützt eine Diagnose bereits die Fallkonzeption, da evidenz-basierte Behandlungsmethoden auf einem empirisch untersuchten Modell der Störung aufbauen. Und drittens ist Information über die Diagnose und über daraus resultierende therapeutische Vorschläge zentraler Bestandteil der informierten $\mathrm{Zu}$ stimmung, die Patient*innen zur Behandlung geben müssen (Persons et al. 2019).

\section{Welche hilfreichen Modelle liefert die Psychotherapieforschung zum Thema?}

In Störungsbereichen wie der Depression, in denen überprüfte Therapiemanuale existieren, können kognitive Verhaltenstherapeut*innen auf einige bewährte ätiologische Erklärungs- und therapeutische Behandlungsmodelle zurückgreifen. Hier werden zwei Ansätze vorrangig genannt (z. B. Hautzinger 2013).
Im „Verstärkungstheoretischen Modell“ von Lewinsohn (1974) wurde die zentrale Rolle von Aktivitäten für die depressive Stimmung beschrieben. Deprimierte Menschen ziehen sich häufig sozial zurück und geben Dinge auf, die ihnen bisher Spaß gemacht haben. Oft haben sie einen schwierigen interaktionellen Stil mit negativen Folgen im zwischenmenschlichen Bereich. Dadurch entsteht eine Abwärtsspirale, in der positive Erfahrungen abnehmen, immer mehr Zeit für negatives Grübeln bleibt und eine Depression entsteht. In der Behandlung wird dem über einen systematischen Aufbau von angenehmen Aktivitäten und Arbeit an zwischenmenschlichen Fertigkeiten entgegengewirkt. Dieser Zugang ist vom Prinzip einfach, empirisch sehr gut überprüft und wirksam (Martell et al. 2010/2015).

Der zweite zentrale Zugang, in dem Bewertungen eine prominente Rolle spielen, ist das Kognitive Modell der Depression von Beck (1967), das mittlerweile auffast alle Bereiche psychischer Störungen ausgedehnt wurde (z.B. Hofmann 2013; Margraf und Schneider 2018a, 2018b). In der Biografie entsteht eine Vulnerabilität („Diathese“) aufgrund von häufigen schwierigen Erfahrungen mit wichtigen Bezugspersonen (Eltern, Großeltern, Lehrer*innen, Gleichaltrige etc.), die zu negativen Grundannahmen über sich selbst, die Umwelt und die Zukunft führt. („Kognitive Triade“; Beck et al. 2010). Durch einen aktuellen Auslöser wird sie aktiviert (,Diathese-Stress-Modell“). Teufelskreise halten die Symptomatik aufrecht. Über „sokratische Gesprächsführung“ und kognitives Umstrukturieren wird an einer Veränderung von dysfunktionalen Gedanken und Überzeugungen gearbeitet.

Für die Fallkonzeption folgt, dass wir bei unserer Patientin auf die Suche gehen nach negativen Annahmen über sich selbst, die Umwelt und die Zukunft, die sich aus ihrer Lebensgeschichte erklären lassen. Die Diathese sollte durch einen erkennbaren Stressor ausgelöst worden sein. Die resultierende dysfunktionale Bewältigung und der Verlust an positiven Erfahrungen sind häufige Teufelskreise, die die Störung aufrechterhalten. Ein depressiver Interaktionsstil kann eine Rolle spielen. Andererseits ist es wichtig, Ressourcen zu identifizieren, die die Patientin bisher geschützt haben und Ziele in der Therapie maßgeblich unterstützen können (Kuyken et al. 2009).

Frau S. beschreibt Rückzugsverhalten mit weniger angenehmen Aktivitäten [Verstärkerverlust] und deutlich mehr Zeit zum Grübeln, das sich bei der Patientin um Hilflosigkeit und Einsamkeit dreht [negative Grundannahmen/Diathese]. Diese beiden Themen begleiten sie schon sehr lange. Frau S. schildert, dass sie mit einer deutlich depressiven Mutter und einem emotional distanzierten $\mathrm{Va}$ ter aufgewachsen sei, die beide wenig verfügbar 
Tab. 1 Horizontale Verhaltensanalyse und Differenzierung der einzelnen Elemente in einem SORKC-Schema anhand des Beispiels einer depressiven Patientin Frau S. (vgl. Knappe und Härtling 2017; Tuschen-Caffier und van Gemmeren 2018)

\begin{tabular}{|c|c|c|}
\hline Stimulus & Interne und externe Auslöser & $\begin{array}{l}\text { Sonntag Nachmittag, der Partner arbeitet schon wieder, Frau S. denkt an Montag, die Arbeit und den stän- } \\
\text { dig fordernden Chef }\end{array}$ \\
\hline Organismus & $\begin{array}{l}\text { Situationsüber-greifende biolo- } \\
\text { gische und psychische Merk- } \\
\text { male der Person }\end{array}$ & $\begin{array}{l}\text { Biologisch: ev. gewisse genetische Prädisposition (depressive Mutter) } \\
\text { Negative Grundannahmen: "Ich bin hilflos.“ „Ich bin allein." } \\
\text { Abgeleitete dysfunktionale Überzeugungen (bedingte Annahmen/Bewältigung): „Andere werden mir nicht } \\
\text { helfen, wenn ich es brauche, ich versuche es erst gar nicht." "Wenn ich zeige, dass ich Hilfe brauche, bin } \\
\text { ich eine Belastung und mein Gegenüber wird sich distanzieren.“ „Ich muss um jeden Preis und immer die } \\
\text { Erwartungen erfüllen, sonst werden sich wichtige Menschen von mir entfernen.“" }\end{array}$ \\
\hline Reaktion & $\begin{array}{l}\text { Problemverhalten bzw. Sym- } \\
\text { ptome auf der kognitiven, } \\
\text { emotionalen, physiologischen } \\
\text { und behavioralen Ebene }\end{array}$ & $\begin{array}{l}\text { Kognitiv: „Ich schaffe das morgen nicht mehr. Ich kann mich nicht wehren. Ich werde meinen Chef enttäu- } \\
\text { schen.“ „Mein Partner ist nicht für mich da. Ich habe niemanden, der mir hilft.“ } \\
\text { Emotional: Hilflosigkeit, tiefe Niedergeschlagenheit } \\
\text { Physiologisch: Anspannung, Erschöpfung } \\
\text { Behavioral: Rückzug auf die Couch, nicht reden, grübeln, stilles Weinen, für sich allein sein }\end{array}$ \\
\hline Kontingenz & $\begin{array}{l}\text { In welcher Form zeigt sich Zu- } \\
\text { sammenhang mit Konsequenz? } \\
\text { manchmal, meistens, immer? }\end{array}$ & Meistens \\
\hline CKonsequenz & $\begin{array}{l}\text { Kurzfristig: Negatives lässt } \\
\text { nach, Positives taucht auf } \\
\text { Langfristig: Negative Konse- } \\
\text { quenzen, die zu Leidensdruck } \\
\text { führen }\end{array}$ & $\begin{array}{l}\text { Kurzfristig (erleichternd oder positiv): etwas Erleichterung durch Rückzug, Weinen und Resignation; be- } \\
\text { fürchtete negative Reaktion des Partners bei Suche nach Unterstützung und die erwartete eigene Enttäu- } \\
\text { schung darüber wurden vermieden } \\
\text { Langfristig (negativ): Selbstwirksamkeit sinkt, negative Annahmen über sich, Partner und Zukunft werden } \\
\text { bestätigt/nicht korrigiert, Beziehung verschlechtert sich, adaptive Bewältigung kann sich nicht entwickeln }\end{array}$ \\
\hline
\end{tabular}

waren. Ihre jüngere, schillernde Schwester habe den Großteil der verbleibenden Aufmerksamkeit bekommen [historische Wurzeln]. Sie selbst sei häufig mit ihren kindlichen Sorgen allein geblieben und hätte die Erfahrung gemacht, dass sie nicht wichtig sei [negative Grundannahmen]. Sie hätte in ihrer sehr ruhigen Art versucht, eine gute Schülerin, unermüdliche Freundin und angepasste Tochter zu sein, die wenig Belastung verursacht. Sie habe sich mit ihren eigenen Bedürfnissen untergeordnet, um die Beziehung zu sichern [Bewältigung abgeleitet aus den Grundannahmen]. Sie erreichte das über Strenge und Selbstdisziplin, die immer wieder auch eine sehr harte innere Kritikerin werden kann [Bewältigung]. Dies sei auch in Partnerschaften ein zentraler Interaktionsstil geblieben [Bewältigung]. Die Unterordnung in der ersten Beziehung machte daher die Trennung des Partners von ihr [Auslöser] zu einer hilflosen Situation des Verlassenwerdens [Symptome].

Durch den Wegfall einer Kollegin vor $6 \mathrm{Mo}$ naten sei die Arbeit merkbar mehr geworden. Sie habe sich ihrem Chef gegenüber nicht abgrenzen können [Auslöser].

Trotz ihrer deutlichen Beeinträchtigungen beschreibt die Patientin, dass sie weiterhin die Anforderungen in der Arbeit erfüllt habe, sie sei sehr verlässlich und könne hartnäckig sein. Sie sei finanziell abgesichert, unterhalte sehr gute Freundschaften, die trotz ihres sozialen Rückzugs wertschätzend und hilfreich seien. Sie ist sportlich und intelligent, sie hat einen ausgezeichneten trockenen Humor und kann in Beziehungen eine angenehme Atmosphäre herstellen [Ressourcen].
In dem Beispiel können eine kognitive Diathese und aktivierende Ereignisse klar identifiziert werden, die eine Veränderung von Erleben und Verhalten bewirkt haben. Die innere Strenge ist meistens Resilienz (als Disziplin), aber auch dysfunktionale Bewältigung (als ewige Kritikerin). Die soziale Kompetenz der Patientin ist eine große Ressource und macht deutlich, dass ein Fertigkeitentraining nicht notwendig ist. Aber sie vermeidet aus Angst zwischenmenschliche Situationen, in denen Klärungsbedarf besteht.

\section{Die individualisierte Fallkonzeption: Vom Allgemeinen zum Besonderen}

Eine prototypische Fallkonzeption macht nur begrenzt das Besondere der Situation von Frau S. sichtbar, das in unserer Schilderung bereits deutlich wird. Sie ist nicht geplagt durch Wertlosigkeitsgefühle, sondern durch Hilflosigkeits- und Einsamkeitsgefühle. Sie bewältigt nicht durch gereizte Konfrontation, Vermeidung oder eine lähmende Opferhaltung, sondern durch Aufopferung, Unterordnung und Leistung. Ihre innere Kritikerin treibt sie hier an und wird immer wertender, um das Letzte aus sich herauszuholen. Wie kommt es dazu, dass die Patientin genau so und nicht anders bewertet und bewältigt? Für eine Erklärung braucht es eine individualisierte Konzeption. Hier werden die zentralen Herangehensweisen der KVT beibehalten, während die Bedeutung der Diagnose abnimmt.

Diese in der Forschung als „generic cognitive model“ (Beck und Haigh 2014) benannte Herangehensweise wurde auch als „case conceptualization-driven approach“ (Persons 2008) bzw. auch als „transdiagnostische KVT“ (Creed et al. 2016) bezeichnet. Dabei 
Abb. 1 In einer individualisierten kognitiv-verhaltenstherapeutischen Fallkonzeption werden Beispiele aus der Problemliste einer horizontalen und vertikalen Verhaltensanalyse unterzogen. In einem knappen "hypothetischen Bedingungsmodell“" wird eine Arbeitshypothese der ätiologischen Faktoren zusammengefasst, die die Grundlage für die Therapieplanung darstellt. Dieser Prozess wird in der Regel mehrfach durchlaufen
Evidenz-basiertes Standardverfahren nicht angemessen/ausreichend?

Individualisierte Fallkonzeption

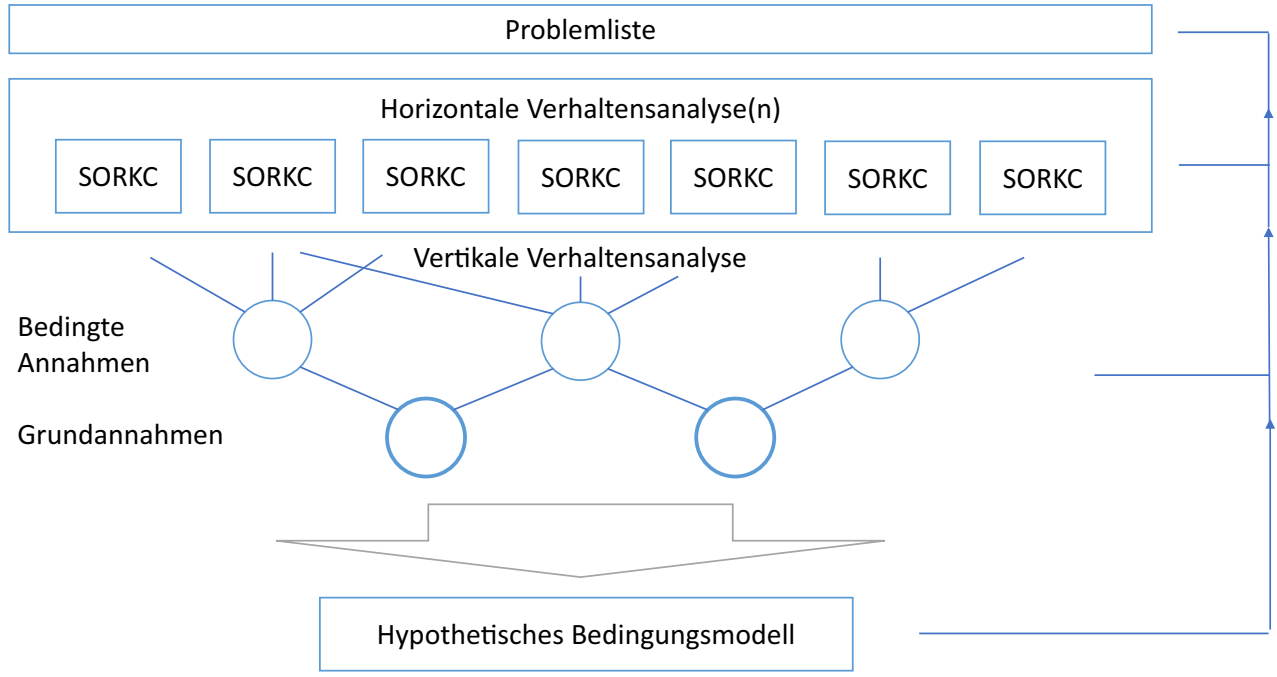

werden Elemente standardisierter Manuale strategisch ausgewählt, um die hypothetischen Mechanismen hinter den Problemen zu verändern (Waltman und Sokol 2017). Dieser Zugang wurde ebenfalls empirisch untersucht (Kuyken et al. 2008).

Der praktische Ausgangspunkt ist zunächst eine Problemliste (Persons 2008), bevor die zentralen psychologischen Mechanismen beschrieben werden, die die Hauptprobleme von Patient*innen auslösen und aufrechterhalten und Ansatzpunkte in der Therapie werden können. Dabei stehen in der KVT mit „horizontalen Verhaltensanalysen“ (sog. SORKC-Schemas, s. oben Tab. 1) und „vertikalen Verhaltensanalysen“ (Identifikation von „bedingten Annahmen“ und „Grundannahmen“; s. unten Abb. 2) differenzierte Methoden zur Verfügung, die das Problemverhalten von Patient*innen auf unterschiedlichen Analyseebenen betrachten (Abb. 1).

\section{Die Problemliste}

Symptome stehen in der Regel auf der Liste der Dinge, die Patient*innen in der Therapie verändern möchten. Die Problemliste umfasst aber auch weitere Bereiche, die mit der Symptomatik überlappen oder relativ unabhängig sein können (gesundheitliche, berufliche, familiäre, partnerschaftliche Probleme etc.). Sie sollte möglichst vollständig sein (Persons 2008). Empirisch betrachtet kommen dabei erfahrene Praktiker*innen bei gleichen Patient*innen zu $60 \%$ bis $70 \%$ zu einer übereinstimmenden Liste, Anfänger*innen nur auf $30 \%$ (Dobson und Dobson 2017).

Frau S. schildert auf ihrer Problemliste zunächst (1) die depressive Symptomatik und (2) die Belas- tung am Arbeitsplatz wegen der nie enden wollenden Anforderungen ihres Chefs. Erst bei näherer Betrachtung des zeitlichen Verlaufs der Symptome wird auch (3) die Qualität des emotionalen Kontakts in der Partnerschaft und (4) die Zukunftsplanung ein Punkt auf der Liste. In ihrer 6-jährigen Partnerschaft könne sie diese Themen nur begrenzt ansprechen, der Partner sei zu sehr mit eigenen beruflichen Problemen beschäftigt. Dies habe sie die ersten Jahre aber nicht zu sehr gestört. Erst im letzten Jahr hätte ihr Bedürfnis, die gemeinsame Familienplanung zu diskutieren, die Schwierigkeiten in der Partnerschaft deutlicher hervorstechen lassen. Hier stünden weitreichende Entscheidungen an. Es zeigt sich (5) ein Muster des Übergehens der eigenen Bedürfnisse und eine für das Gegenüber sehr angenehme, sich unterordnende Beziehungsgestaltung (auch in weiteren Bereichen einschließlich der Therapie), bei der

(6) die eigene Gestaltungskraft und Spontaneität auf der Strecke bleibt.

Wenn die Problemliste gemeinsam erschöpfend geklärt ist, sind wiederholte horizontale und vertikale Verhaltensanalysen (VA) der nächste Schritt, um Hypothesen zu den Mechanismen dahinter zu formulieren (vgl. Abb. 1). Eine gute therapeutische Beziehung - getragen von einer Haltung des „kollaborativen Empirismus“ (Kazantzis et al. 2017) - ist Voraussetzung, d.h. gemeinsames Arbeiten und Entdecken mit einem Geist der wissenschaftlichen Neugier und des Experimentierens. 
Abb. 2 Beispielhafter Ausschnitt aus einer vertikalen Verhaltensanalyse der depressiven Patientin Frau S. mit zwei zentralen Grundannahmen

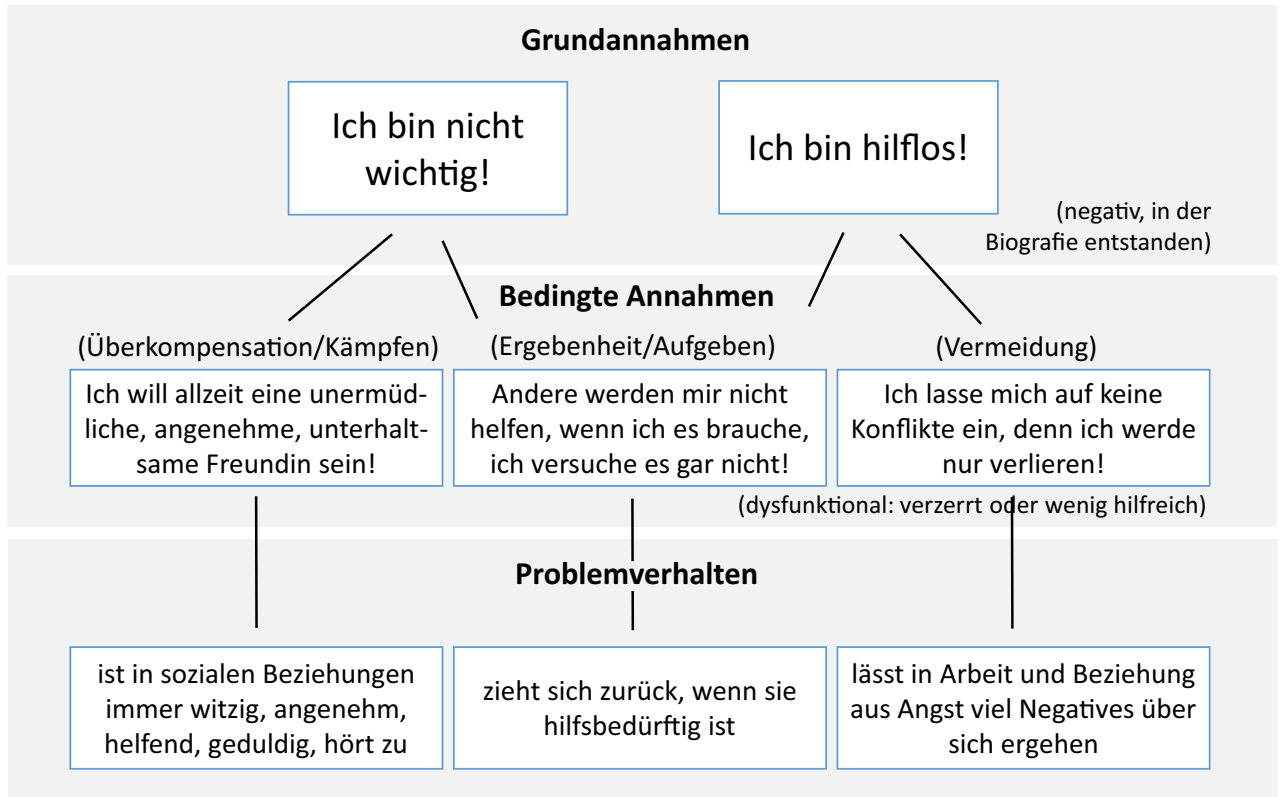

\section{Die horizontale Verhaltensanalyse: Probleme unter der Lupe}

Kanfer et al. (2012) beschreiben die horizontale VA als ein Herauszoomen eines Verhaltensausschnitts, der dann über ein sog. SORKC-Schema (Kanfer und Saslow 1969/1974) beschrieben wird. Dieses ursprünglich rein lerntheoretische Schema wurde später um dysfunktionale Kognitionen wie Überzeugungen, Gedanken und innere Bilder erweitert (z.B. Kanfer et al. 2012; Knappe und Härtling 2017; Tuschen-Caffier und van Gemmeren 2018). Es sei an dieser Stelle angemerkt, dass diese klassische Notation eine Besonderheit der deutschsprachigen KVT darstellt und international nur selten verwendet wird (z.B. Rizvi 2019).

Ein Beispiel eines SORKC-Schemas mit Bezeichnung und Bedeutung der einzelnen Elemente ist in Tab. 1 dargestellt. Es ist die formale Aufschlüsselung von hypothetisch angenommenen vorausgehenden und nachfolgenden Bedingungen eines konkreten Problemverhaltens, die in einem ungünstigen Teufelskreis münden. Die horizontale VA ist also eine Hypothese über funktionale Zusammenhänge: Wie genau landet eine Person in einer depressiven Stimmung und kommt dort nicht mehr heraus?

Frau S. schildert ihre stärksten depressiven Stimmungen abends und am Wochenende, häufig Sonntag am späten Nachmittag, wenn der Partner bereits wieder Arbeiten für die kommende Woche begonnen habe. Sie selbst habe Gedanken und Bilder an die am Montag auf sie zukommenden Arbeiten und ihren Chef, der ständig Neues von ihr fordere. Angesichts dieser inneren Bilder sinke ihre Stimmung, sie sehe sich selbst nicht in der Lage, an dieser Situation etwas zu ändern. Sie fühle sich überfordert, erschöpft und schutzbedürftig. Der Partner habe kaum Kapazität und Verständnis für ihre Situation, zumal sie in der Regel kaum von ihren Belastungen erzähle. Sie sehe sich nicht in der Lage, Unterstützung von ihm zu holen oder gar einzufordern. Sie fühle sich neben ihm und mit ihm zutiefst einsam, werde weinerlich und leer und ziehe sich auf die Couch zurück und versuche gleichzeitig, sich möglichst wenig anmerken zu lassen.

Die Aufschlüsselung des Ablaufs beginnt mit einer Beschreibung des Problemverhaltens R, bevor vorausgehende und nachfolgende Bedingungen mit funktionaler Bedeutung identifiziert werden (Rizvi 2019; vgl. Tab. 1).

Die horizontale Verhaltensanalyse liefert eine erste funktionale Hypothese dazu, aus welchen Gründen die Patientin in einer hilflosen Situation verharrt, obwohl es vielleicht adaptivere Alternativen in der Bewältigung gäbe. Zunächst wird deutlich, dass die Qualität der Partnerschaft eine größere Rolle in der Symptomatik spielt als zunächst angenommen. Kurzfristig ist tatsächlich ein gewisser Nutzen im depressiven Verhalten erkennbar. Es werden schwierige und bedrohliche Schritte (in der Arbeit und in der Partnerschaft) und weitere Enttäuschungen vermieden. Langfristig wird das Problem dadurch nicht gelöst, sondern verschlimmert sich.

Diese horizontale VA wird grundsätzlich mit jedem Problemverhalten der Problemliste wiederholt und kollaborativ durchgeführt. So können über gemeinsame Grundannahmen und die daraus abgeleitete Bewältigung hierarchisch übergeordnete Ziele sichtbar werden, die weiter in einer vertikalen VA untersucht und integriert werden. Ohne diese Integration hät- 
ten wir viele horizontale VAs ohne Bezug zueinander (Kanfer et al. 2012; vgl. Abb. 1).

\section{Die vertikale Verhaltensanalyse: Zusammenschau und Identifikation von Mechanismen}

In der O-Variable in Tab. 1 findet sich bereits jener erste Teil von Kognitionen, der in der vertikalen VA das depressive Verhalten der Patientin erklären hilft. Weiteres Problemverhalten wird möglicherweise durch ähnliche Überzeugungen gesteuert (ist also „funktional äquivalent“; Caspar 2018). Es können aber auch weitere „Grundannahmen“ und „bedingte Annahmen“ (s. unten Abb. 2) sein, die sie in diesen Situationen antreiben. Die Analyse und Integration dieser Kognitionen ist das Ziel der vertikalen VA, die mit biografischen Erfahrungen logisch im Einklang stehen muss.

Schließlich entstehen Grundannahmen über sich, die Umwelt und die Zukunft nicht aus heiterem Himmel, sondern sind langjährige und häufig völlig automatisierte Haltungen, die aus unzähligen (bei Patient*innen negativen) Erfahrungen mit wichtigen Bezugspersonen resultieren. Die Anamnese liefert zentrale Informationen, die erhärten können, warum eine Grundannahme entstanden ist. J. Beck (2013) hat drei zentrale Bereiche negativer Grundannahmen präzisiert. Demnach lassen sich bei Patient*innen die Bereiche (1) „Bindung/Alleinsein“, (2) „Kontrolle/Hilflosigkeit“ und (3) „Selbstwert/Minderwertigkeit“ identifizieren. Diese Themen können einzeln oder in Kombination das Erleben einer Person bestimmen. Sie korrespondieren mit universellen Grundbedürfnissen (z. B. Grawe 2000; Abb. 2).

Im Umgang mit eigenen Grundannahmen bzw. negativen Emotionen sucht die betroffene Person Wege, um Grundbedürfnisse zu befriedigen oder sich wenigstens $\mathrm{zu}$ schützen und die negativen Gefühle $\mathrm{zu}$ lindern. Abgeleitete Ziele, Regeln und Pläne werden als „bedingte Annahmen“ bezeichnet, weil sie Ableitungen von der Grundannahme darstellen, daher bedingt sind. Andererseits werden sie als „vermittelnde Annahmen" bezeichnet, weil sie zwischen Grundannahmen und dem konkreten Verhalten in Situationen vermitteln (Beck 2013). Während grundsätzlich eine sehr große Anzahl von derartigen Überzeugungen in einer Person wahrnehmbar ist, werden wir nur jene in eine therapeutisch hilfreiche vertikale VA einbinden, die Problemverhalten erklären. Diese Überzeugungen sind notwendigerweise dysfunktional (verzerrt oder nicht hilfreich; Dobson und Dobson 2017) und sind im kognitiven Modell verantwortlich dafür, dass Personen krankheitswertige Störungen entwickeln. Die sog. „Plananalyse“ (Caspar 2018), die wiederum vorwiegend im deutschsprachigen Raum bekannt ist, spielt sich vollständig auf der Ebene der bedingten Annahmen ab.
Eine hilfreiche Differenzierung kommt aus der „Schematherapie“ von Young (Young et al. 2003/2005), in der (1) Schema-Vermeidung, (2) Schema-Ergebenheit und (3) Schema-Überkompensation als sehr unterschiedlich erscheinende Bewältigungsstrategien ein und desselben Schemas beschrieben werden. Auch bei Frau S. sind ihre Bewältigungsversuche diesen Strategien zuordenbar. Die Darstellung einer vertikalen Verhaltensanalyse wird häufig grafisch versucht, um die Zusammenhänge der verschiedenen Komponenten deutlich zu machen. Diese Analyse ist ebenfalls das Ergebnis einer Kollaboration zwischen Patient*in und Therapeut*in (Abb. 2).

In Abb. 2 ist erkennbar, dass in zwei von drei Bereichen negative Grundannahmen gemeinsam identifiziert werden konnten. Frau S. versucht seit ihrer Kindheit, in Beziehungen außerhalb ihrer Familie wichtig zu sein (Überkompensation/Kämpfen), sie ist resigniert, wenn es um Hilfe bei ihren eigenen Bedürfnissen geht (Ergebenheit) Aufgeben) und vermeidet Konflikte, weil sie vermeintlich keine Chance hat (Vermeidung). Sie hat in der Überkompensation wertvolle Ressourcen entwickelt, allerdings sind diese immer wieder gepaart mit dem Zurückhalten eigener Bedürfnisse, was bereits zu unausgewogenen Beziehungen geführt hat und sich auch in der Therapie bemerkbar machen könnte. Sie könnte durch ihre sehr zurückhaltende Art der Beziehungsgestaltung eine besonders angenehme Patientin sein wollen. Gleichzeitig würde sich therapeutischer Fortschritt wohl genau dann bemerkbar machen, wenn sie es sich erlaubt, hier weniger angenehm zu sein!

Die vertikale VA erlaubt eine wertvolle Integration von verschiedenen Mustern von Patient*innen (z.B. wichtig sein für andere, Bindung sichern, Kontrolle behalten), sie kann also zeigen, was zusammengehört, funktional äquivalent ist (Caspar 2018). Genauso kann sie aber auch deutlich machen, dass manche Verhaltensweisen von Patient*innen oberflächlich ähnlich aussehen, obwohl sie unterschiedlichen Funktionen dienen. Nett zu sein auch in schwierigen Beziehungen kann bei Frau S. sowohl Ausdruck einer von ihr energisch verfolgten Überkompensation (Ich will eine angenehme Gesprächspartnerin sein!) als auch Ausdruck eines Vermeidens von Konflikten aus Angst vor Bindungsverlust sein.

Eine gute vertikale VA liefert ausgesprochen viel Information über die Mechanismen, die bei Patient*innen für Probleme verantwortlich sind und langjährige Teufelskreise und innere Konflikte produzieren können. Dabei ist ein Ziel die maximale Reduktion von Information. Sie liefert damit gleichzeitig äußerst wertvolle Informationen für die Behandlungsplanung. Identifizierte Teufelskreise können über "Verhaltensexperimente" untersucht und verändert werden (z. B. das Experiment, ihrem Freund 
ihre Stimmungen mitzuteilen und seine und ihre eigenen Reaktionen $\mathrm{zu}$ beobachten und ihre eigenen Vorhersagen bzw. Befürchtungen damit systematisch $\mathrm{zu}$ überprüfen). Bedingte Annahmen und Grundannahmen werden Ansatzpunkte für kognitive und schematherapeutische Interventionen (Persons 2008).

Durch die hohe Komplexität der Aufgabe und das $\mathrm{Maß}$ an Abstraktionsarbeit ist das Ergebnis für die Patient*innen in der therapeutischen Arbeit nicht leicht zu behalten. Deshalb wird für die gemeinsame Arbeit ein griffiges maximal vereinfachtes Gesamtbild der Problematik von Patient*innen gezeichnet, ein Narrativ, in dem die wesentlichen ätiologischen Faktoren zusammengefasst sind und die Folgen für das therapeutische Arbeiten erkennbar und ableitbar sind (Bruch 2015): das hypothetische Bedingungsmodell ${ }^{1}$. Es ist der dritte und letzte Teil der individualisierten Fallkonzeption. Ihre möglichst knappe Zusammenfassung ist die Basis für die Therapieplanung.

\section{Hypothetisches Bedingungsmodell: Theory in a nutshell}

Das hypothetische Bedingungsmodell (HBM) bleibt immer hypothetisch, es ist eine kollaborativ entwickelte Arbeitshypothese $\mathrm{zu}$ den Problemen von $\mathrm{Pa}-$ tient*innen auf der Basis des vorhandenen Wissens, die jederzeit revidiert werden kann und muss, wenn sich durch zusätzliche Informationen neue oder angemessenere Perspektiven ergeben (Kanfer et al. 2012; Reinecker 2015). Müssen mehrere Probleme und Störungsbereiche in das Gesamtbild integriert werden (was eher die Regel ist), dann wird jener Störungsbereich in den Vordergrund gestellt, der primär ist, die Hauptbelastung darstellt oder für das aktuelle Aufsuchen der Psychotherapie verantwortlich ist. Wenn das HBM angemessen formuliert wurde, erlaubt es gewisse Vorhersagen im Erleben und Verhalten von Patient*innen und erleichtert empathisches Einfühlen von Therapeut*innen (Persons 2008). Es ist praktikabel, wenn es unmittelbar Ansatzpunkte für therapeutische Veränderung erkennen lässt. Und es sollte auch auf mögliche Schwierigkeiten im therapeutischen Prozess frühzeitig hinweisen, indem motivationale oder interaktionelle Besonderheiten der Person deutlich werden, die die therapeutische Beziehung und Zusammenarbeit (positiv oder negativ) beeinflussen können. Dieses Modell ist schließlich eine wesentliche Basis für transparentes therapeutisches Vorgehen (als zentrales Merkmal der KVT) und die informierte Zustimmung zur Behandlung (Persons et al. 2019).

Das HBM besteht üblicherweise aus 3 Teilen, die inhaltlich bereits beschrieben wurden: (1) Genese und Beschreibung der Vulnerabilität/Diathese, (2) Auslöser/Stressoren in der jüngeren Vergangenheit, die

\footnotetext{
1 Wir reservieren diesen Begriff für das Gesamtbild der Probleme von Patient*innen, auch wenn jede horizontale und vertikale VA bereits als ein (Teil-)HBM betrachtet werden kann.
}

die Diathese aktiviert haben und zu Symptomen geführt haben und (3) aufrechterhaltende Bedingungen, Teufelskreise, die möglicherweise kurzfristig Erleichterung verschaffen, aber langfristig negative Konsequenzen haben und Leidensdruck erzeugen. Diese drei Teile werden so aufbereitet und zusammengefasst, dass sie für Patient*innen verständlich sind. Dabei gilt als übergeordnetes Prinzip, das Modell so sparsam wie möglich und so komplex wie nötig zu konzipieren (Eells 2015). Es ist der große Plan, ein grobkörniges Gesamtbild, das jederzeit bei Bedarf mit den horizontalen VAs um fallweise notwendige Details angereichert werden kann.

Genese/Vulnerabilität: Sowohl die Depression der Mutter als auch die emotionale Abwesenheit des Vaters haben Frau S. überzeugend vermittelt, dass sie bei der Bewältigung ihrer Probleme und ihren emotionalen Bedürfnissen auf sich gestellt ist. Die Vulnerabilität ist entsprechend gekennzeichnet durch negative Grundannahmen, nicht so wichtig/allein und hilflos zu sein. Annehmen der negativen Situation, ohne sich zu wehren, Unterordnen und Sichern von Beziehungen durch Zurückstellen eigener Bedürfnisse, Vermeiden von Konflikten und besonderes Engagement für Freundinnen sind schon in der Kindheit zentrale Bewältigungsstrategien geworden. Ihre sozialen Kompetenzen und Beziehungen haben sie lange Zeit effektiv vor depressiven Einbrüchen geschützt und sind auch heute zentrale Ressourcen. Die Patientin sieht sich aber als Belastung, wenn sie Bedürfnisse äußert. Entsprechend versucht sie ihre Probleme selbst $z u$ lösen bzw. zieht sich zurück, wenn es ihr schlecht geht.

Auslöser: Die erste depressive Episode wurde ausgelöst durch die unkontrollierbare Trennungssituation vom Ex-Freund. Der zweite Stressor, der die Bewältigung überlastet und die Diathese aktiviert, ist einerseits das aktuelle Beziehungsproblem, das durch die ungeklärte Familienplanung sichtbar wird, und andererseits die verstärkte Anforderung am Arbeitsplatz, die nicht durch Abgrenzung reguliert werden kann und nochmals deutlich die Belastung erhöht.

Aufrechterhaltende Bedingungen: Zum einen versucht die Patientin trotz Überforderung weiterzuarbeiten, ohne auf die eigenen Grenzen zu achten. Sie vermeidet Konflikte mit dem Chef, dessen Erwartungen sie unbedingt erfüllen möchte. Außerdem sieht sie sich nicht in der Lage, emotionale Erleichterung zu schaffen. In der Partnerschaft akzeptiert sie die distanzierte Seite ihres Partners seit Beginn der Beziehung. Ihre emotionalen Bedürfnisse durch die Überlastung am Arbeitsplatz lassen sie befürchten, sowohl beim Partner wie auch bei den Freundinnen eine Belastung zu sein und damit die Beziehungen zu verschlechtern. Also tut sie nichts und sie bekommt nichts. 
Der Rückzug und das damit verbundene negative Grübeln reduzieren positive Erfahrungen und verstärken die Symptomatik und die Belastung. Gleichzeitig bleiben ihre negativen Überzeugungen und Grundannahmen damit so, wie sie sind, und werden nicht korrigiert, sondern sogar weiter verstärkt.

Die Fallkonzeption wird mit Patient*innen immer wieder diskutiert, schriftlich festgehalten und verfeinert, wenn sich neue Prozesse erkennen lassen. Dabei werden Patient*innen explizit eingeladen, Zweifel oder Unklarheiten zu äußern, bis ein beidseitig akzeptables Modell entwickelt ist (Hautzinger 2013; Kazantzis et al. 2017) und Maßnahmen für das therapeutische Vorgehen abgeleitet werden können. In unserem Fallkonzept lassen sich Verhaltensaktivierung und Aufbau von angenehmen Aktivitäten als wichtige Schritte erkennen, um die negativen Folgen des Rückzugs zu reduzieren. Es macht deutlich, dass die Patientin eine Reihe von problematischen, biografisch sehr nachvollziehbaren Bewältigungsstrategien aufgebaut hat, die in Verhaltensexperimenten überprüft werden könnten (z. B. sich Freundinnen zu öffnen und anhand von beobachtbaren Indikatoren zu überprüfen, ob sie von diesen tatsächlich als Belastung wahrgenommen wird). Es könnten manche Einstellungen über sokratische Gesprächsführung disputiert werden, die sie etwa davor zurückhalten, in der Beziehung emotionale Unterstützung einzufordern oder in der Arbeit notwendige Grenzen zu ziehen. Schließlich ist es eine längere Aufgabe, die jahrzehntealten negativen Grundannahmen immer wieder aufzuweichen und damit die zentrale Diathese für die Störung schrittweise zu verändern und auf diese Weise zukünftigen Rückfällen vorzubeugen. Dabei ist die Gestaltung der therapeutischen Beziehung ein wichtiger Faktor. Die Patientin ist in der Therapie „pflegeleicht“ und angenehm, es wäre verführerisch, eine „einfache“ Patientin vor sich zu sehen. Im Gegenteil erlaubt das Fallkonzept die Funktion ihres zwischenmenschlichen Verhaltens zu erkennen, das zweifelsohne eine große Ressource darstellt, aber gleichzeitig immer wieder weiter fortsetzt, dass eigene Bedürfnisse und Selbstbehauptung auf der Strecke bleiben. Ein Aussteigen aus diesem Muster, ein Fördern von Spontaneität, Eigenständigkeit und zunehmender Selbstvalidierung könnten in der Therapie ihren Anfang nehmen, wenn das HBM therapeutisches Handeln leitet. Dabei wird die Angst vor Beziehungsabbruch und Alleinsein eine Reihe von erwartbaren „Widerständen“ in der Therapie aktivieren, die mit dem HBM ebenfalls validierend identifiziert werden können.

Eine logisch konsistente Fallkonzeption, die - ausgehend von empirisch abgesicherten Modellen - die einzigartige Person in ihrer Überforderung, ihren Ressourcen und ihrem (auch kulturellen) Kontext erklären hilft und so die Gestaltung von Interventionen und die therapeutische Beziehungsgestaltung leitet, sieht am Ende einfach, knapp und klar aus. Gleichzeitig ist sie wahrscheinlich eine der komplexesten Aufgaben in der Kognitiven Verhaltenstherapie.

Funding Open access funding provided by University of Innsbruck and Medical University of Innsbruck.

Interessenkonflikt H. Mitmansgruber, A. Fahlböck, A. Fink, L. Mäni Kogler, I. Müller, A. Painold, C. Streicher-Pehböck und G. Gatterer geben an, dass kein Interessenkonflikt besteht.

Open Access Dieser Artikel wird unter der Creative Commons Namensnennung 4.0 International Lizenz veröffentlicht, welche die Nutzung, Vervielfältigung, Bearbeitung, Verbreitung und Wiedergabe in jeglichem Medium und Format erlaubt, sofern Sie den/die ursprünglichen Autor(en) und die Quelle ordnungsgemäß nennen, einen Link zur Creative Commons Lizenz beifügen und angeben, ob Änderungen vorgenommen wurden.

Die in diesem Artikel enthaltenen Bilder und sonstiges Drittmaterial unterliegen ebenfalls der genannten Creative Commons Lizenz, sofern sich aus der Abbildungslegende nichts anderes ergibt. Sofern das betreffende Material nicht unter der genannten Creative Commons Lizenz steht und die betreffende Handlung nicht nach gesetzlichen Vorschriften erlaubt ist, ist für die oben aufgeführten Weiterverwendungen des Materials die Einwilligung des jeweiligen Rechteinhabers einzuholen.

Weitere Details zur Lizenz entnehmen Sie bitte der Lizenzinformation auf http://creativecommons.org/licenses/by/4. $0 /$ deed.de.

\section{Literatur}

American Psychological Association (2020). Psychological treatments. https://www.div12.org/psychologicaltreatments/.Zugegriffen:29. Febr. 2020.

Bartling, G., Echelmeyer, L., \& Engberding, M. (2016). Problemanalyse im psychotherapeutischen Prozess: Leitfaden für die Praxis (6. Aufl.). Stuttgart: Kohlhammer.

Beck, A. T. (1967). Depression: Causes and treatment. Philadelphia: University of Pennsylvania Press.

Beck, J. (2013). Praxis der Kognitiven Verhaltenstherapie (2.Aufl.).Weinheim: Beltz.

Beck, A. T., \& Haigh, E. A. P. (2014). Advances in cognitive theory and therapy: the generic cognitive model. Annual Review of Clinical Psychology, 10, 1-24.

Beck, A. T., Rush, A. J., Shaw, B. F., \& Emery, G. (2010). Kognitive Therapie der Depression. Weinheim: Beltz.

Bruch, M. (2015). Beyond diagnosis: case formulation in cognitive behavioural therapy (2. Aufl.). Chichester:Wiley.

Caspar, F. (2018). Beziehungen und Probleme verstehen (4.Aufl.). Göttingen:Hogrefe.

Creed, T. A., Frankel, S., German, R., et al. (2016). Implementation of transdiagnostic cognitive therapy in community behavioural health: a Beck community initiative. Journal of Consulting and Clinical Psychology, 84, 1116-1126.

Dimidjian, S. (Hrsg.). (2019). Evidence-based practice in action: bridging clinical science and intervention. New York: Guilford.

Dobson, D., \& Dobson, K.S. (Hrsg.). (2017). Handbook of cognitive-behavioral therapy(2.Aufl.). NewYork:Guilford.

Eells, T. D. (2007). Handbook of psychotherapy case formulation (2. Aufl.). NewYork: Guilford.

Eells, T. D. (2015). Psychotherapy case formulation. Washington, DC:American Psychological Association. 
Grawe, K. (2000). Psychologische Therapie. Göttingen:Hogrefe. Hautzinger, M. (2013). Kognitive Verhaltenstherapie bei Depressionen (7.Aufl.). Weinheim: Beltz.

Hofmann, S. G. (2013). The Wiley handbook of cognitive-behavioral therapy. NewYork: Wiley-Blackwell.

Kanfer, \& Saslow (1974). Verhaltenstheoretische Diagnostik. In D. Schulte (Hrsg.), Diagnostik in der Verhaltenstherapie (S. 24-59). München: Urban \& Schwarzenberg.

Kanfer, F. H., Reinecker, H., \& Schmelzer, D. (2012). Selbstmanagement-Therapie (5.Aufl.). Berlin: Springer.

Kazantzis, N., Deane, F. P., Ronan, K. R., \&L'Abate, L. (2017). The therapeutic relationship in cognitive-behavioral therapy: a clinician's guide. NewYork: Guilford.

Key, B., \& Bieling, P. J. (2014). Beyond DSM diagnosis: the pros and cons of cognitive case formulation. In G.P. Brown \& D. A. Clark (Hrsg.), Assessment in cognitive therapy (S.221-239). NewYork: Guilford.

Knappe, S., \& Härtling, S. (2017). Diagnostik und Verhaltensanalyse. Weinheim: Beltz.

Kuyken, W., Padesky, C.A., \& Dudley, R. (2008). The science and practice of case conceptualization. Behavioural and Cognitive Psychotherapy, 36, 757-768.

Kuyken, W., Padesky, C.A., \& Dudley, R. (2009). Collaborative case conceptualization: working effectively with clients in cognitive-behavioral therapy. NewYork: Guilford.

Ledley, D. R., Marx, B.P., \& Heimberg, R.G. (2018). Making cognitive behavioural therapy work: clinical processes for new practitioners. NewYork: Guilford.

Lewinsohn, P. M. (1974). A behavioural approach to depression.InR. J.Friedman \& M. M. Katz (Hrsg.), The psychology of depression (S. 157-178). NewYork: Wiley.

Lilienfeld, S. O., Ritschel, L.A., Lynn, S. J., \& Latzman, R.D. (2019). The insufficiently appreciated raison d'etre of evidence-based practice. In S. Dimidjian (Hrsg.), Evidencebased practice in action: Bridging clinical science and intervention (S. 43-63). New York: Guilford.

Margraf, J., \& Schneider, S. (Hrsg.). (2018a). Grundlagen, Diagnostik, Verfahren und Rahmenbedingungen psychologischer Therapie (4. Aufl.). Bd. 1. Berlin: Springer.

Margraf, J., \& Schneider, S. (Hrsg.). (2018b). Psychologische Therapie bei Indikationen im Erwachsenenalter(4.Aufl.). Bd.2. Berlin:Springer.
Martell,C. R.,Dimidjian, S., \&Herman-Dunn, R. (2015). Verhaltensaktivierung bei Depression. Stuttgart: Kohlhammer.

National Institute of Clinical Excellence, NICE (2020). Improving health and social care through evidence-based guidance. https://www.nice.org.uk/. Zugegriffen: 29. Febr. 2020 .

Needleman, L. D. (1999). Cognitive case conceptualization: $a$ guidebook for practitioners. New York: Routledge.

Nezu, A. M., Nezu, C. M., \& Lombardo, E. (2004). Cognitive-behavioral case formulation and treatment design: a problem-solving approach. NewYork: Springer.

Persons, J. B. (2008). The case formulation approach to cognitive-behavior therapy. NewYork: Guilford.

Persons, J. B., Brown, C. L., \& Diamond, A. (2019). Case formulation-driven cognitive-behavioral therapy. In D. Dobson \& K.S. Dobson (Hrsg.), Handbook of Cognitive-Behavioral Therapy (2.Aufl. S. 145-168). New York: Guilford.

Reinecker, H. (2015). Verhaltensanalyse: Ein Praxisleitfaden. Göttingen: Hogrefe.

Rizvi, S. L. (2019). Chain analysis in dialectical behavior therapy. New York: Guilford.

Sturmey, P. (Hrsg.). (2009). Clinical case formulation: Varietes of approaches. New York: Wiley-Blackwell.

Tarrier, N. (2006). Case formulation in cognitive behaviour therapy: the treatment of challenging and complex cases. London: Routledge.

Tuschen-Caffier, B., \& van Gemmeren, B. (2018). Problemund Verhaltensanalyse. In J. Margraf \& S. Schneider (Hrsg.), Grundlagen, Diagnostik, Verfahren und Rahmenbedingungen psychologischer Therapie 4. Aufl. Lehrbuch der Verhaltenstherapie, (Bd. 1, S. 287-297). Berlin: Springer.

Waltman, S. H., \& Sokol, L. (2017). The generic model of cognitive behavioral therapy: a case conceptualization-driven approach. In S. G. Hofmann \& J. G. Amundson (Hrsg.), The science of cognitive behavioral therapy (S.3-17). London: Academic Press.

Young, J. E., Klosko, J. S., \& Weishaar, M. E. (2005). Schematherapie: Ein praxisorientiertes Handbuch. Paderborn: Junfermann.

Hinweis des Verlags Der Verlag bleibt in Hinblick auf geografische Zuordnungen und Gebietsbezeichnungen in veröffentlichten Karten und Institutsadressen neutral. 